\title{
The development of Negative Self-Beliefs Inventory (NSBI): Cultural adaptation and psychometric validation
}

Xiaoqing Tang, Wenjie Duan, Ying Wang, Pengfei Guo

Social anxiety is an emotional disorder common to various populations around the world. The newly developed Self-Beliefs Related to Social Anxiety Scale (SBSA) aims to assess three kinds of self-beliefs through 15 items that include self-related cognitive factors that evidently result in social anxiety. This study explored the psychometric characteristics of SBSA among 978 Chinese. An eight-item Negative Self-beliefs Inventory (NSBI) was developed through qualitative and quantitative analyses. Exploratory factor analysis, confirmatory factor analysis, and multi-group confirmatory factor analysis suggested that NSBI contained clear, meaningful, stable, and invariant three-factor structure consistent with the original SBSA. Further analyses showed that the three subscales and the entire scale exhibited high internal consistency (0.779-0.837), good criterion validity, and good convergent and divergent validity (i.e., negative associations with flourishing and positive associations with anxiety, depression, and stress). These findings indicated that NSBI is reliable and valid for measuring negative self-beliefs in the Chinese population. Higher total score of NSBI indicates the more serious negative self-beliefs. Limitations of the present study and implications for research and practice were also discussed. Further studies are needed to evaluate the predictive ability, incremental validity, and potential role of NSBI in clinical and large-scale populations. 
Abstract

2 Social anxiety is an emotional disorder common to various populations around the world. The

3 newly developed Self-Beliefs Related to Social Anxiety Scale (SBSA) aims to assess three kinds

4 of self-beliefs through 15 items that include self-related cognitive factors that evidently result in

5 social anxiety. This study explored the psychometric characteristics of SBSA among 978

6 Chinese. An eight-item Negative Self-beliefs Inventory (NSBI) was developed through

7 qualitative and quantitative analyses. Exploratory factor analysis, confirmatory factor analysis,

8 and multi-group confirmatory factor analysis suggested that NSBI contained clear, meaningful,

9 stable, and invariant three-factor structure consistent with the original SBSA. Further analyses

10 showed that the three subscales and the entire scale exhibited high internal consistency $(0.779-$

11 0.837), good criterion validity, and good convergent and divergent validity (i.e., negative

12 associations with flourishing and positive associations with anxiety, depression, and stress).

13 These findings indicated that NSBI is reliable and valid for measuring negative self-beliefs in the

14 Chinese population. Higher total score of NSBI indicates the more serious negative self-beliefs.

15 Limitations of the present study and implications for research and practice were also discussed.

16 Further studies are needed to evaluate the predictive ability, incremental validity, and potential

17 role of NSBI in clinical and large-scale populations.

18 Keywords: self-beliefs, reliability, validity, measurement invariance, Negative Self-Beliefs

19 Inventory 
20 Title: The Development of Negative Self-Beliefs Inventory (NSBI): Cultural Adaptation and

21 Psychometric Validation

22

23

24

25

26

27

28

29

\section{Introduction}

Mild anxiety or discomfort experienced by individuals when speaking in public or social situations is a normal psychological reaction. However, when the anxiety or discomfort causes severe distress and impairs normal social functioning, it may evolve into a mood disorder called social anxiety disorder. Social anxiety is one of the major emotional disorders that is characterized by remarkable and persistent fear of negative evaluation in social related contexts (American Psychiatric Association 2013). In Western countries, the lifetime prevalence of social anxiety disorder was estimated at $12.10 \%$ to $13.00 \%$ among different adult populations (Furmark 2002; Kessler et al. 2005; Polo et al. 2011). Very few studies have investigated the prevalence of social anxiety disorder among the general Chinese adult population (Hofmann et al. 2010). A recent large-scale research revealed that the 12-month and lifetime prevalence of social anxiety disorder among 11,527 Chinese military personnel were $3.34 \%$ and $6.22 \%$, respectively (Wang et al. 2014). The low prevalence in China, as with other psychiatric disorders (e.g., depression, Smith 2014), may be partly attributed to culture-related low detection rate (Hofmann et al. 2010; Smith 2014).

Hofmann et al. (2010) examined the cultural factors related to social anxiety and concluded that the degree of expression of social anxiety depends on the social norms, cultural background, and ethnic/racial characteristics. Accordingly, culture-related factors need to be carefully considered in different countries when conduct social anxiety-related assessment and treatment (Hofmann et al. 2010). For instance, Caldwell-Harris \& Ayçiçegi (2006) investigated the effect of individualism-and-collectivism on the reported psychological distress in both 
43 individualism and collectivism countries. The results indicated that the respondents living in

44 collectivism countries usually reported low symptoms on anxiety, depression, schizophrenia, and

45 antisocial personality disorder (Caldwell-Harris \& Ayçiçegi 2006). Another study further found

46 that the participants in collectivistic cultures showed higher social anxiety levels and more

47 positive attitude to socially avoidant behaviors. These findings implied that it is important and

48 meaningful to conduct cultural adaptation when applied western (i.e., individualism countries)

49 social anxiety inventories into eastern countries (i.e., collectivism countries).

50 Self-Beliefs of Social Anxiety

51 Determining the risk factors of social anxiety is very important in developing intervention

52 programs and psychotherapies. Ng et al. (2014) conducted a systematic review of 17 evidence-

53 based studies and identified that negatively perceived self-related information (e.g., negative

54 self-imagery) is the key cognitive factor that increases social anxiety in both clinical and non-

55 clinical populations, as had been emphasized in different cognitive models of social anxiety

56 (Clark \& Wells 1995; Hofmann 2007; Rapee \& Heimberg 1997). For instance, Clark \& Wells

57 (1995) stated that individuals' excessive attention to internal negative thoughts, feelings, and

58 physical sensations in social contexts would confirm their perceived negative impression and

59 beliefs of themselves that, in turn, would increase the level of anxiety. Similarly, Rapee \&

60 Heimberg (1997) and Hofmann (2007) recognized that social anxiety results from a discrepancy

61 between individuals' negatively perceived self-related information and the assumed audiences'

62 high expectation. Therefore, these cognitive models of social anxiety and cognitive-behavior

63 therapies suggest that a reduction of negative self-related beliefs would positively relieve social

64 anxiety. 

developed the Self-Beliefs Related to Social Anxiety Scale (SBSA), which measures three types

67 of self-beliefs in social contexts proposed by Clark \& Wells (1995). The scale consists 15 items

68 (i.e., four items for high standard beliefs on social performance, HSB; seven items for conditional beliefs on social evaluation, $\mathrm{CB}$; and four items for unconditional beliefs on the self, UB). Preliminary psychometric evaluation demonstrated that the scale displays excellent reliability (i.e., Cronbach's alpha $>$.82), satisfactory item-item and item-total correlations (i.e.,

Pearson correlations ranged from .72 to .89), meaningful factor structure, good convergent and divergent validity, and acceptable incremental and discriminative validity (Wong \& Moulds 2009; Wong \& Moulds 2011; Wong et al. 2014). Nevertheless, the stable of the factor structure was unclear. Wong \& Moulds (2011) revealed a two-factor structure (i.e., CB and UB merged into one factor, and HSB was the other factor) in exploratory factor analysis using 600 nonclinical undergraduates, whereas the following confirmatory factor analysis (CFA) demonstrated that the three-factor structure exhibited better fit than the two-factor model. Finally, they adopted the three-factor model (Wong \& Moulds 2011) that consistent with the Clark \& Wells (1995)'s

80 theoretical model. Recently, Heeren et al. (2014) likewise examined the structural validity of

81 SBSA among a French-speaking community sample. Their study utilized CFA and revealed its 82 replicable three-factor structure, good reliability, and concurrent validity. It should be noted that social anxiety-related assessment are culturally dependent

84 (Hofmann et al. 2010). Equivalence of concepts and inventory items should be evaluated and 85 adjusted before the western culture-based measurement can be applied into eastern countries.

\section{Equivalence of Inventories in Different Cultures}



interpretations of the items, which were referred to as functional equivalence and conceptual equivalence of items in previous studies (Cheung et al. 2011; Duan et al. 2012). The aforementioned studies were also conducted in Western countries. No study has yet examined the factor structure and psychometric characteristics of SBSA in Eastern cultures, hence there is a need to examine the cognitive understanding of each item in the context of Chinese culture and to re-explore and validate the factor structure.

Previous studies suggested that cultural adaptation should be considered to ensure equivalence of inventories in different cultures (Ho et al. 2014b). Specifically, Johnson (1998) proposed that cross-cultural equivalence of inventories should be obtained through four kinds of equivalences, namely, linguistic, conceptual, metric, and functional equivalence. Linguistic equivalence refers to the linguistic accuracy of each item in different cultures and emphasizes quality of translation. Conceptual equivalence refers to similarity in participants' understanding of factors and concepts despite coming from different cultures. Metric equivalence avoids the floor and/or ceiling effects. Finally, functional equivalence indicates that the behavior and/or thoughts described by the items are the same in different cultural contexts (Ho et al. 2014a; Ho et al. 2014b; Johnson 1998). Traditionally, translation and back-translation, as well as confirmatory factor analysis have been recognized as the most commonly used approaches in cross-cultural psychometric evaluation studies. Nevertheless, several scholars (e.g., Hui \& Triandis 1985; Kankaraš \& Moors

107 2010) argued that only part linguistic and conceptual equivalence could be obtained through the 108 aforementioned traditional steps, and that the equality of translations, cultural relevance, 109 measurement equivalent of constructs, and validity of the adapted instrument need to be 
110 additionally and carefully considered. The World Healh Organization (2011) published a four-

111 step guideline for refining the original "translation and back-translation" method, which

112 emphasized the role of experts in moderating the equality of translations and partly compose the

113 deficiencies of traditional approach. Metric and functional equivalences can often be explored

114 through qualitative methods, such as group interview. However, very few researchers have done

115 so. Our previous experience demonstrated that the combination of these rules and methods is

116 helpful in ensuring the equivalence of measurement tools in different cultures. For instance,

117 Values in Action Inventory of Strengths (VIA-IS) is a widely used measurement for assessing

118 character strengths among diverse populations by using 240 items (Peterson \& Seligman 2004).

119 However, the factor structures of the VIA-IS are various in different countries (Duan et al. 2012).

120 Further qualitative and quantitative inspections found that several items were common social

121 expectations that may lack sensitivity; several items may not be appropriate in representing the

122 spirituality-related culture in the Mainland China; and several items may represent socially

123 unacceptable behavior in Mainland China (cf. Duan et al. 2012; Ho et al. 2014a). After these

124 inappropriate items have been deleted, a cultural fit and stable strength-structure was obtained

125 (Duan et al. 2013).

126 The Present Study

127 We expect to achieve several objectives, namely to (1) obtain equality between the

128 English and Chinese translation and examine the cultural relevance of each item through

129 qualitative and quantitative analyses; (2) validate the Chinese version of SBSA and test its factor

130 structure, reliability, criterion validity, convergent validity, and divergent validity; specifically,

131 SBSA should show positive relations with trait anxiety, state anxiety, social anxiety, stress, and

132 depression; whereas show negative relations with psychological wellbeing (e.g., Flourishing); (3) 
133 establish the cross-gender measurement invariance for meaningful comparisons between

134 different groups, which can guarantee similar latent constructs across groups (Vandenberg \&

135 Lance 2000); and (4) to obtain solid psychometric evidence through a short form that is practical

136 and convenient to apply in the community, clinical, and large-scale settings for purposes of

137 research and intervention evaluation (Ziegler et al. 2014).

\section{Method}

\section{Participants and Procedures}

A total of 978 (428 males, 550 females; $M=20.73, S D=3.46)$ participants from six

141 different universities were involved in this quantitative survey. Those universities are located in

142 Eastern, Central, and Western China; this distribution is helpful in balancing the economic and

143 social background of the participants. Participants with active physical and mental illnesses were

144 excluded. No participant reported serious medical history and long-term medication. The

145 participants were asked to provide written informed consent before completing the

146 questionnaires. The Institutional Review Board of the Southwest University approved this study.

147 The entire sample was divided into four independent subsamples; each subsample

148 completed a distinct questionnaire package created for specific research purposes to control the

149 source of common-method bias (Podsakoff et al. 2003; Podsakoff et al. 2012) and reduce

150 participants' cognitive load and fatigue (Rammstedt \& Beierlein 2014). Subsample $1\left(\mathrm{n}_{1}=330\right.$;

151171 males, 159 females; $M=20.42, S D=0.77)$ completed the Chinese version of SBSA for

152 exploratory factor analysis; subsample $2\left(\mathrm{n}_{2}=330 ; 164\right.$ males, 166 females; $M=20.40, S D=$

153 0.73) also completed the Chinese version of SBSA but for confirmatory factor analysis;

154 subsample $3\left(\mathrm{n}_{3}=155 ; 44\right.$ males, 111 females; $\left.M=21.45, S D=6.12\right)$ completed the short form

155 of SBSA, Liebowitz Social Anxiety Scale, and State-Trait Anxiety Inventory for examining 
156 criterion validities; and subsample $4\left(\mathrm{n}_{4}=163 ; 49\right.$ males, 114 females; $\left.M=21.38, S D=0.73\right)$

157 completed the short form of SBSA, Depression Anxiety Stress Scales, and Flourishing Scale for

158 examining convergent and divergent validities.

159 After the investigation was completed, the study objective and corresponding

160 interpretations were explained to the participants. Data were collected from May to November in 1612014.

\section{Measurements}

Self-Beliefs Related to Social Anxiety (SBSA). SBSA is a 15-item self-reporting

164 questionnaire that assesses the strengths of self-perceived beliefs related to the self in social

165 contexts (Wong \& Moulds 2009; Wong \& Moulds 2011; Wong et al. 2014). It contains three

166 subscales (four-item HSB, seven-item CB, and four-item UB). Participants were asked to rate

167 each item on an 11-point Likert scale from 0 (do not agree at all) to 10 (strongly agree). Subscale

168 scores and total scores were calculated by summing up the scores of the corresponding items.

169 High scores reflect the strong strengths of self-beliefs.

170 Liebowitz Social Anxiety Scale (LSAS). LSAS is a 24-item self-reporting scale that

171 measures anxiety and the avoidance of various social performances and situations (Liebowitz

172 1987). For each social performance and situation, participants were required to rate their feelings

173 and behaviors on a four-point Likert scale ranging from 0 (never) to 3 (always). High scores of

174 the total scale indicate increased levels of social anxiety. The Chinese version of LSAS

175 processed good psychometric properties among both clinical and non-clinical populations (He \&

176 Zhang 2004). The Cronbach's alpha of the current sample is .934.

177 State-Trait Anxiety Inventory (STAI). STAI is a widely used self-reporting inventory

178 for assessing the state (20 items) and trait (20 items) of anxiety among diverse populations 
179 (Spielberger et al. 1970). Different instructions for the two subscales were provided to guide

180 participants in giving appropriate responses. All items were rated on a four-point Likert scale

181 ranging from 0 (never) to 3 (very obvious/always). The scores of two subscales were summed

182 separately; high scores reflected increased levels of state anxiety or trait anxiety. The Cronbach's

183 alpha of the state and trait subscales in the current sample are .893 and .847 , respectively.

184 Depression Anxiety Stress Scales (DASS). Depression, anxiety, and stress over the past

185 week were assessed through a short version of DASS, which is a 21 -item self-reporting scale that

186 contains three subscales (seven items per subscale) (Lovibond \& Lovibond 1995). Previous

187 studies revealed its good internal consistency and factor structure (e.g., Antony et al. 1998). High

188 scores of the three subscales separately reflect high level or severity of depression or anxiety.

189 The Cronbach's alpha of the current study is .859 .

190 Flourishing Scale (FS). FS is a new inventory that assesses the important aspects of the

191 functioning of human functioning through eight items (Diener et al., 2010), which reflects the

192 general psychological wellbeing of individuals. Participants used a seven-point Likert scale to

193 evaluate the items by using 1 (strongly disagree) to 7 (strongly agree). A high mean score of the

194 whole scale indicates a high degree of psychological wellbeing. Tang et al. (2014) demonstrated

195 its good psychometric characteristics among the Chinese. The Cronbach's alpha of the current

196 sample is .789.

197 Translation of SBSA

198 The steps recommended by Hambleton et al. (2004) and Sperber (2004) are

199 comprehensively considered to achieve linguistic equivalence in the present study. The first

200 author of this manuscript established a triangular group, including one $\mathrm{PhD}$ student majoring in

201 Psychology, one PhD student majoring in Sociology, and one psychology professor who severed 
202 as moderator. All members are bilingual experts (i.e., English and Chinese). First, the original

203 SBSA was translated into Chinese by the psychology PhD student. The sociology PhD student

204 then back-translated the Chinese version of SBSA into English. The professor supervised the

205 entire translation process and was responsible for verifying the conformity of the translated

206 English items with the original ones, as well as the precision of the Chinese items. Discrepancies

207 were discussed thoroughly and revised by the triangular group and the first author.

208 Data Analysis Plan

209 Data analysis was composed of both qualitative and quantitative stages.

210 The qualitative stage aimed to conduct cognitive interview among undergraduates to

211 obtain feedback regarding the appropriateness and meanings of the SBSA items in the context of

212 Chinese culture. Previous studies (e.g., Duan et al. 2012) suggested that cognitive feedback from

213 the target group would be helpful in refining the translations and/or determining what culturally

214 inappropriate items to delete. The first author conducted interviews among 20 undergraduates

215 who were unaware of the purpose of the study and had not attended the quantitative survey. Four

216 types of standardized questions, which were used in previous studies, were presented to them

217 (Duan et al. 2012): (1) Please tell me whether you understand this item or not. What do you think

218 the item is asking? (2) What did you think about when you first read this item? (3) Do you

219 understand the description of response choices in the questionnaire? What is the meaning of

220 "strongly agree"? (And so on for other responses) Which one do you choose? Why? (4) Could

221 you select a response choice that reflects your true opinion of this item? Why? Questions (1) and

222 (2) assessed conceptual and functional equivalence, whereas Questions (3) and (4) assessed

223 metric equivalence. 
225 (i.e., Cronbach's alpha) by using the first subsample. Items that could improve the internal 226 consistency coefficient when deleted were considered to be removed. As what the original 227 authors did (Wong \& Moulds 2009), maximum likelihood factor analysis with promax rotation 228 method was adopted to evaluate the factor structure. Confirmatory factor analysis and multi229 group confirmatory factor analysis were conducted using the second subsample to identify the 230 best-fit model and evaluate measurement invariance across genders. A short form with high 231 factor loadings, clear factor structure, and measurement invariance across different gender 232 groups was expected to be developed based on the above steps.

234 further tested using the third and fourth subsamples. Pearson correlations were calculated 235 between the short form and similar psychological variables (e.g., trait anxiety, state anxiety, and 236 social anxiety), psychological distress (e.g., depression, stress, and anxiety), and psychological 237 wellbeing (e.g., flourishing).

238 Data were analyzed using SPSS 21.0 and Mplus 7.0.

\section{Results}

\section{Cognitive Interview}

The results of cognitive feedback revealed that several items in the 15-item SBSA might

242 contain conceptual and functional issues, but no metric issue was proposed. Most participants $243(\mathrm{n}=18)$ indicated that Item 4 "I have to appear intelligent and witty" and Item 7 "I have to convey 244 a favorable impression" described strategies of impression management in Chinese culture rather 245 than self-beliefs because the information conveyed by the items met Chinese social expectations. 246 In other words, individuals in front of other people are always prone to impress and show their 
247 good side. Accordingly, Item 4 and Item 7 are "positive behaviors" and "advisable beliefs",

248 rather than the negative beliefs related to anxiety in Mainland China. Most of the participants

249 would rate highly on the two items. In addition, more than half of the respondents $(\mathrm{n}=12)$

250 considered Item 3 "If people do not accept me, I'm worthless" and Item 5 "If someone does not

251 like me, it must be my fault" possibly refers to high-standard self-beliefs, which indicate that an

252 individual should be valuable and get people to like him/her. In other words, Items 3 and 5 may

253 be conceptually varied in Western and Eastern societies. Additionally, several participants $(\mathrm{n}=$

254 9) thought that Item 1 "If I make mistakes, others will reject me" had uncertain meaning,

255 especially with regard to the word of "mistake". The severity of the mistakes would affect their

256 rating of this item. For instance, some students said that if the mistake was really small or only

257 related to him/her selves, then others would not reject them; on the other hand, if the mistake was

258 really matter or impaired damaged the collective interest, then others would reject them. Thus the

259 "mistake" may contain different meanings to different individuals. Several students $(n=14)$ did

260 not understand why anxiety would be a sign of weakness (Item 8 "If people know I'm anxious,

261 they will think I'm weak."). Accordingly, we assumed that these items (i.e., Items 1, 3, 5, 4, 7,

262 and 8) might lack sensitivity of assessment in Chinese culture, and that their removal will

263 improve the scale's reliability and validity. Nevertheless, it should be noted that the items are not

264 directly removed based on the above cognitive interview results. Both qualitative and

265 quantitative results should be considered before the removing of the items.

266 Internal Consistency

267 The Cronbach's alpha of the original 15-item scale was 0.880 . However, the results

268 suggested that the alpha would increase to .888 if Item 4 was deleted. After Item 4 has been

269 removed, the results again suggested that the removal of Item 7 would increase the Cronbach's 
270 alpha to 891 . Integrating the results of cognitive interviews, Items 4 and 7 were removed from

271 the 15 -item pool.

272 Exploratory Factor Analysis

273 Maximum likelihood factor analysis with promax rotation method was performed among

274 the remaining 13 items. $\mathrm{KMO}=.895$ and Bartlett's Test of Sphericity $=1978.376(p<.001)$

275 indicated that the current data pool was appropriate for analysis. Three factors were extracted, 276 but several items were cross-loaded. For instance, Item 1 was loaded on factors 2 (loading $=$ 277.487 ) and 3 (loading = .465); thus, Item 1 was removed. After several explorations, Items 2, 3, 5, 278 and 8 were removed as cross-loadings. The removed items were likewise questionable, as 279 reflected by the cognitive interviews. Finally, eight items were left (i.e., items 6, 9, 10, 11, 12, 28013,14 , and 15) for the final factor analysis. The result indicated that the eight-item pool 281 remained appropriate for factor analysis $(\mathrm{KMO}=.851$; Bartlett's Test of Sphericity $=1263.272$, $282 p<.001$ ), and a clear three-factor structure was obtained (Table 1). All factor loadings of the 283 eight items were higher than .67. Considering the content validity of the revised inventor with 284 regards to the original scale, the shortened scale was renamed as the Negative Self-beliefs 285 Inventory (NSBI). The Cronbach's alpha of the HSB subscale in the NSBI was .779, that of the 286 CB subscale was .784, and that of the UB subscale was .837. These results indicated that the 287 internal consistency of the NSBI was good (Maydeu-Olivares et al. 2007).

\section{Confirmatory Factor Analysis}

Subsample $2\left(\mathrm{n}_{2}=330\right)$ was used to further investigate the factor structure of NSBI 290 through confirmatory factor analysis. Comparative Fit Index (CFI > .95), and Root-mean-square 291 Error of Approximation (RMSEA $<.50$ or .80 ) were adopted to evaluate the comparable models 292 and/or structural equation models (Hu \& Bentler 1999). Following three previous studies 
293 (Heeren et al. 2014; Wong \& Moulds 2009; Wong \& Moulds 2011; Wong et al. 2014), three 294 comparable models were constructed, including a three-factor model, a two-factor model (i.e.,

295 the items of CB subscale and UB subscale loaded on the same factor), and a single-factor model 296 (i.e., all items loaded on one factor). The goodness-of-fit indices of the three models are shown

297 in Table 2 and suggest that the three-factor model achieved the best fit in our sample.

298 Standardized path coefficients of the three-factor model are shown in Figure 1 and are significant 299 at .001 levels. All standardized item loadings were higher than .710. These results supported the 300 three-factor structure of NSBI among the Chinese undergraduate population.

\section{Multi-group Confirmatory Factor Analysis} Meredith (1993) and Byrne (2012) recommended that measurement invariance could be

303 achieved by examining the four levels of equivalence from the weakest to the strongest,

304 including configural invariance, weak/metric factorial invariance, strong/scalar factorial

305 invariance, and the variance of the means of latent variables. Two criteria were used to determine 306 whether equivalence was maintained between a more restricted model and a less restricted one, 307 including the change in CFI $(\triangle \mathrm{CFI})$ and change in RMSEA ( $\triangle \mathrm{RMSEA})$. Researchers suggested 308 that $|\Delta \mathrm{CFI}|<.010$ (Cheung \& Rensvold 2002) and $|\Delta \mathrm{RMSEA}|<.015$ (Chen 2007) supported the 309 equivalence of measurement. Chen (2007) considered $|\Delta \mathrm{RMSEA}|$ as an important supplement 310 indicator when the total sample size was larger than 300 , as with the current one $\left(\mathrm{n}_{2}=330\right)$. The 311 results presented in Table 3 reveal acceptable changes in the CFI and RMSEA, which supported

312 the measurement equivalence of NSBI in the different gender groups.

\section{Criterion Validity}

314 Criterion validity was examined using the third subsample $\left(n_{3}=155\right)$. Pearson correlation

315 results are shown in Table 4. In addition to the HSB subscale, the CB subscale, UB subscale, and 
316 total scale of NSBI were positively related $(r=.160-.357)$ to other anxiety-related

317 measurements, including state anxiety, trait anxiety, and LSAS. Among the three subscales of

318 NSBI, the UB subscale displayed the highest correlation coefficients.

\section{Convergent and Divergent Validity}

320 Convergent and divergent validities were examined by calculating the Pearson

321 correlations between NSBI and both the negative and positive psychological outcomes. As 322 expected, all subscales and the entire scale exhibited negative relations with flourishing $(r=-$

323.108 to -.303$)$ and positive relations with depression, anxiety, and stress $(r=.235-.414)$ (Table

324 5). All correlation coefficients were significant at .001 levels with the exception of the HSB 325 subscale.

\section{Discussion}

The aim of this study was to validate the culturally adapted SBSA. Through a series of statistical analysis, an eight-item NSBI was developed and was proven to be capable of providing stable and clear three-factor structure, acceptable reliability, good criterion, convergent, and divergent validity.

A total of seven items (items 1, 2, 3, 4, 5, 7, and 8) were removed from the original 15item pool (Wong \& Moulds 2009) through cognitive interview and exploratory factor analyses. Previous related studies also found that several of these items were questionable. For instance, in

334 the deleted items, Heeren et al. (2014) indicated that the item loadings of Items 1, 4, and 7 in the

335 French version were lower than .40. Similarly, Items 4 and 7 exhibited the lowest item loadings

336 among all four items of the HSB subscale, and Item 2 was the lowest among all items of UB

337 subscale (Wong et al. 2014). The removal of these low loading items improved the factor

338 structure to some extent. Actually, both the removing and remaining items highlighted the role of 
339 self-construals in cross-cultural social anxiety studies, which defined how people relate to others

340 and the social context (Hofmann et al. 2010). The removed items were all related to independent

341 self-construals (e.g., Item 4 "I have to appear intelligent and witty" and Item 7 "I have to convey

342 a favorable impression"), which were frequent in western countries or individualistic societies

343 and reflected the tendency of viewing self as autonomous from the social context; whereas the

344 remaining items were all related to interdependent self-construals (e.g. Item 6 "People think

345 badly of me" and Item 10 "If I don't get everything right, I'll be rejected"), which were common

346 in eastern countries or collectivist societies and reflected the tendency of viewing self as being

347 integrated with others and social context (Hofmann et al. 2010).

348 In addition, as discussed previously, a two-factor structure from exploratory factor

349 analysis was against the three-factor structure from confirmatory factor analysis (Wong \&

350 Moulds 2011). After deleting several cross-loading items, the three-factor structure was clearly

351 obtained through exploratory factor analysis and further validated through exploratory factor

352 analysis (using another independent sample). Thus, the results supported the possibility of the

353 cross-loading phenomenon as the cause of inconsistency in the results of Wong \& Moulds (2011)

354 and Wong \& Moulds (2009). Reports from the revised eight-item NSBI also preliminarily

355 revealed measurement equivalence across gender groups. The overall fit of the four levels of

356 invariance models was acceptable, which means that indicators (i.e., items) load on similar

357 factors with equal factor loadings across different groups (Bontempo \& Hofer 2007), and that the

358 corresponding factor intercepts and latent mean differences were equivalent across genders.

359 Thus, meaningful comparisons of the three factors of NSBI can be made in different gender

360 groups (Vandenberg \& Lance 2000). 
362 reflected social anxiety-related beliefs and a low correlation with state and trait anxiety.

363 However, the current study obtained unexpected results; SBSA-SF had a high correlation with

364 general anxiety (i.e., state and trait anxiety), and relative low correlation with LASA. This result

365 was likewise found in a French-speaking sample (Heeren et al. 2014). We considered the lack of

366 a clinical sample of social anxiety as the reason behind the above results because this study

367 recruited college students who may not exhibit high scores on social anxiety as participants. In

368 terms of relationship between NSBI and state/trait anxiety, UB subscale exhibited the highest

369 correlations with state/trait anxiety among the three subscales. The negative evaluation reflected

370 by the UB subscale was recognized as a trait-like vulnerability (Chase et al. 2010; Clark 2002;

371 Weeks \& Howell 2012), which was associated with a wide range of emotional disorders (e.g.,

372 general anxiety, social anxiety, and depression). This association also explains why NSBI was

373 associated with depression in the present study as well as in previous ones (Heeren et al. 2014;

374 Wong et al. 2014). NSBI was also negatively related to flourishing and positively related to

375 stress. All of these results indicate acceptable convergent and discriminant validities.

376 Many studies have found similar cognitive patterns and co-occurrences between

377 individuals with social phobia and depressive disorders (Dozois \& Frewen 2006; Wittchen \&

378 Fehm 2001). This observation could be another possible explanation as to why NSBI was

379 associated with anxiety and depression. Numerous studies demonstrated that rumination was a

380 cognitive trigger of depression, and reduced rumination thinking had a positive effect on

381 depressive symptoms (e.g., Smith \& Alloy 2009; Zawadzki et al. 2013). If the self-beliefs

382 assessed by NSBI were important cognitive factors of social anxiety, and if these self-beliefs

383 differed with rumination in conceptual and functional levels, then we can further hypothesize 
384 that rumination, compared to self-beliefs related to social anxiety, occupies incremental validity

385 when predicting depression, and that compared to ruminations, self-beliefs related to social

386 anxiety occupy incremental validity when predicting social anxiety. Verifying the above

387 hypotheses and clarifying the relationship between rumination and self-beliefs require further

388 examination through a longitudinal research design and clinical samples in the future.

389 Several limitations of this study should be identified. The major limitation of this study is

390 the use of university student sample and did not involve clinical participants. Hence, this

391 sampling limits the generalizability of the results to all Chinese adults. Furthermore, some of the

392 items being removed in the cognitive debriefing may indicate their immaturity about the social

393 norm and society expectation in Chinese context. Future studies should re-examine the reliability

394 and validity of NSBI with a clinical sample of people suffering from social anxiety disorder

395 and/or a community sample. Second, limited validities were examined in the current study.

396 Future studies should examine whether NSBI exhibits incremental validities when compared to

397 other factors (e.g., rumination) in predicting social anxiety. Third, longitudinal studies should be

398 conducted to clarify the mediation role of NSBI before a meaningful intervention program can be

399 developed. Finally, the short version of SBSA was obtained among the Chinese population.

400 Although the short form of the scale was effective and timesaving in large-scale social surveys

401 (Rammstedt \& Beierlein 2014), psychometric evaluations in other countries, especially in

402 Western countries, should be evaluated further.

403 Our findings indicate that an eight-item Negative Self-beliefs Inventory (NSBI) provides

404 reliable and valid observations on three kinds of maladaptive self-beliefs (Clark \& Wells 1995).

405 According to the above findings, NSBI is related to psychological distress including depression, 
406 anxiety, and stress. A higher total score of NSBI reflects more serious negative self-beliefs, 407 which in turn associates with higher level of psychological distress.

408 
410 American Psychiatric Association. 2013. Diagnostic and statistical manual of mental disorders.

411 Arlington, VA: American Psychiatric Association.

412 Antony MM, Bieling PJ, Cox BJ, Enns MW, and Swinson RP. 1998. Psychometric properties of 413 the 42-item and 21-item versions of the Depression Anxiety Stress Scales in clinical 414 groups and a community sample. Psychological Assessment 10:176-181.

415 Bontempo DE, and Hofer SM. 2007. Assessing Factorial Invariance in Cross-sectional and Longitudinal Studies. In: Ong AD, and Dulmen Mv, eds. Oxford handbook of methods in positive psychology. Oxford, England: Oxford University Press, 153-175.

Byrne BM. 2012. Structureal Equation Modeling with Mplus: Basic Concepts, Application, and Programming. New York: Routledge.

Caldwell-Harris CL, and Ayçiçegi A. 2006. When personality and culture clash: The psychological distress of allocentrics in an individualist culture and idiocentrics in a

Chase HW, Camille N, Michael A, Bullmore ET, Robbins TW, and Sahakian BJ. 2010. Regret collectivist culture. Transcultural psychiatry 43:331-361. and the negative evaluation of decision outcomes in major depression. Cognitive, Affective, \& Behavioral Neuroscience 10:406-413.

Chen FF. 2007. Sensitivity of goodness of fit indexes to lack of measurement invariance. Structural equation modeling 14:464-504.

Cheung FM, van de Vijver FJ, and Leong FT. 2011. Toward a new approach to the study of personality in culture. American Psychologist 66:593-603.

Cheung GW, and Rensvold RB. 2002. Evaluating goodness-of-fit indexes for testing measurement invariance. Structural equation modeling 9:233-255. 
432 Clark DA. 2002. The persistent problem of negative cognition in anxiety and depression: New 433 perspectives and old controversies. Behavior Therapy 32:3-12.

434 Clark DM, and Wells A. 1995. A cognitive model of social phobia. In: Heimberg RG, Liebowitz 435 MR, Hope DA, and Schneier FR, eds. Social phobia: Diagnosis, assessment, and 436 treatment. New York: Guilford Press, 69-93.

437 Dozois DJ, and Frewen PA. 2006. Specificity of cognitive structure in depression and social 438 phobia: A comparison of interpersonal and achievement content. Journal of affective $439 \quad$ disorders 90:101-109.

440 Duan W, Ho SMY, Bai Y, and Tang X. 2013. Psychometric evaluation of the Chinese Virtues 441 Questionnaire. Research on Social Work Practice 23:336-345.

Duan W, Ho SMY, Bai Y, Tang X, Zhang Y, Li T, and Yuen T. 2012. Factor structure of the 443 Chinese Virtues Questionnaire. Research on Social Work Practice 22:680-688.

444 Furmark T. 2002. Social phobia: overview of community surveys. Acta psychiatrica 445 scandinavica 105:84-93.

Hambleton RK, Merenda PF, and Spielberger CD. 2004. Adapting educational and psychological tests for cross-cultural assessment. Hillsdale, NJ, USA: Erlbaum.

He Y, and Zhang M. 2004. Psychometric Investigation of Liebowitz Social Anxiety Scale. Journal of Diagnostics: Concepts and Practice 3:89-93. Validity of the Self-Beliefs in Social Anxiety Scale (SBSA): Adaptation and Validation in a French-Speaking Community Sample. Canadian Journal of Behavioural Science / Revue canadienne des sciences du comportement 46:506-513. 
454 Ho SMY, Duan W, and Tang SCM. 2014a. The psychology of virtue and happiness in western

455

456

457

458

460

461

462

463

464

465

466

467

468

469

470

471

472

473

474

and asian thought. In: Snow NE, and Trivigno FV, eds. The Philosophy and Psychology of Character and Happiness. New York: Routledge, 215-238.

Ho SMY, Rochelle TLR, Law LSC, Duan W, Bai Y, Shih S-M, and Wang G-L. 2014b. Methodological issues in positive psychology research with diverse populations: Exploring strengths among Chinese adults. In: Pedrotti JT, and Edwards LM, eds. Perspectives on the Intersection of Multiculturalism \& Positive Psychology. New York, N.Y.: Springer Science + Business Media B.V., 45-57.

Hofmann SG. 2007. Cognitive factors that maintain social anxiety disorder: A comprehensive model and its treatment implications. Cognitive behaviour therapy 36:193-209.

Hofmann SG, Asnaani A, and Hinton DE. 2010. Cultural aspects in social anxiety and social anxiety disorder. Depress Anxiety 27:1117-1127.

Hu Lt, and Bentler PM. 1999. Cutoff criteria for fit indexes in covariance structure analysis: Conventional criteria versus new alternatives. Structural Equation Modeling: A Multidisciplinary Journal 6:1-55.

Hui CH, and Triandis HC. 1985. Measurement in Cross-Cultural Psychology A Review and Comparison of Strategies. Journal of Cross-Cultural Psychology 16:131-152.

Johnson TP. 1998. Approaches to equivalence in cross-cultural and cross-national survey research. ZUMA-Nachrichten spezial 3:1-40.

Kankaraš M, and Moors G. 2010. Researching measurement equivalence in cross-cultural studies. Psihologija 43:121-136. 
475 Kessler RC, Berglund P, Demler O, Jin R, Merikangas KR, and Walters EE. 2005. Lifetime 476 Prevalence and Age-of-Onset Distributions of DSM-IV Disorders in the National Comorbidity Survey Replication. Archives of general psychiatry 62:593-602.

478

Liebowitz MR. 1987. Social phobia. Modern problems of pharmacopsychiatry 22:141-173.

Lovibond SH, and Lovibond PF. 1995. Manual for the Depression Anxiety and Stress Scales. Sydney, New South Wales, Australia: Psychological Foundation.

Maydeu-Olivares A, Coffman DL, and Hartmann WM. 2007. Asymptotically distribution-free (ADF) interval estimation of coefficient alpha. Psychological methods 12:157-176.

Meredith W. 1993. Measurement invariance, factor analysis and factorial invariance. Psychometrika 58:525-543.

Ng AS, Abbott MJ, and Hunt C. 2014. The effect of self-imagery on symptoms and processes in social anxiety: A systematic review. Clinical Psychology Review 34:620-633.

Peterson C, and Seligman MEP. 2004. Character strengths and virtues: A handbook and classification: Oxford University Press, USA.

Podsakoff PM, MacKenzie SB, Lee JY, and Podsakoff NP. 2003. Common method biases in behavioral research: a critical review of the literature and recommended remedies. Journal of Applied Psychology 88:879.

Podsakoff PM, MacKenzie SB, and Podsakoff NP. 2012. Sources of method bias in social science research and recommendations on how to control it. Annu Rev Psychol 63:539569.

Polo A, Alegría M, Chen C-N, and Blanco C. 2011. The Prevalence, Comorbidity, and Age of Onset of Social Anxiety Disorder among US Latinos. The Journal of clinical psychiatry 72:1096-1105. 
Rammstedt B, and Beierlein C. 2014. Can’t We Make It Any Shorter? Journal of Individual Differences 35:212-220.

Rapee RM, and Heimberg RG. 1997. A cognitive-behavioral model of anxiety in social phobia. Behav Res Ther 35:741-756.

Smith JM, and Alloy LB. 2009. A roadmap to rumination: A review of the definition, assessment, and conceptualization of this multifaceted construct. Clinical Psychology Review 29:116-128.

Smith K. 2014. Mental health: a world of depression. Nature 515:181.

Sperber AD. 2004. Translation and validation of study instruments for cross-cultural research. Gastroenterology 126:S124-S128.

Spielberger CD, Gorsuch RL, and Lushene RE. 1970. Manual for the state-trait anxiety inventory. Palo Alto, CA, USA: Consulting Psychologists Press.

Tang X, Duan W, Wang Z, and Liu T. 2014. Psychometric Evaluation of the Simplified Chinese Version of Flourishing Scale. Research on Social Work Practice:Advance online publication.

Vandenberg RJ, and Lance CE. 2000. A review and synthesis of the measurement invariance literature: Suggestions, practices, and recommendations for organizational research. Organizational research methods 3:4-70.

Wang H, Zhang R, Chen Y, Wang H, Zhang Y, Gan J, Zhang L, and Tan Q. 2014. Social anxiety disorder in the Chinese military: Prevalence, comorbidities, impairment, and treatmentseeking. Psychiatry research 220:903-908. 
519 Weeks JW, and Howell AN. 2012. The bivalent fear of evaluation model of social anxiety:

520 further integrating findings on fears of positive and negative evaluation. Cognitive $521 \quad$ behaviour therapy 41:83-95.

522 Wittchen H-U, and Fehm L. 2001. Epidemiology, patterns of comorbidity, and associated 523 disabilities of social phobia. Psychiatric Clinics of North America 24:617-641.

524 Wong QJ, and Moulds ML. 2009. Impact of rumination versus distraction on anxiety and 525 maladaptive self-beliefs in socially anxious individuals. Behav Res Ther 47:861-867.

526 Wong QJ, and Moulds ML. 2011. Erratum to: a new measure of the maladaptive self-beliefs in social anxiety: psychometric properties in a non-clinical sample. Journal of Psychopathology and Behavioral Assessment 33:285-297.

529 Wong QJ, Moulds ML, and Rapee RM. 2014. Validation of the Self-Beliefs Related to Social 530 Anxiety Scale A Replication and Extension. Assessment 21:300-311.

531 World Healh Organization. 2011. Process of translation and adaptation of instruments. Available at http://www.who.int/substance_abuse/research_tools/translation/en/(accessed Jan 9 2015).

Zawadzki MJ, Graham JE, and Gerin W. 2013. Rumination and anxiety mediate the effect of loneliness on depressed mood and sleep quality in college students. Health Psychology $32: 212-222$.

Ziegler M, Kemper CJ, and Kruyen P. 2014. Short Scales - Five Misunderstandings and Ways to 539 540 


\section{Table 1 (on next page)}

Table 1 Maximum Likelihood Factor Analysis of the Negative Self-beliefs Inventory. $\left(n_{1}=\right.$ 330)

Note. $\mathrm{UB}=$ Unconditional beliefs about self; $\mathrm{CB}=$ Conditional beliefs concerning social evaluation; $\mathrm{HSB}=$ High standards for social performance. 
1 Table 1

2 Maximum Likelihood Factor Analysis of the Negative Self-beliefs Inventory. $\left(n_{1}=330\right)$

Factor

Items

UB CB HSB

Item 9 人们认为我是差劲的 [People think I'm inferior] $\quad .856$

Item

人们不尊重我 [People don't respect me]

.784

14

Item 6 人们认为我很糟糕 [People think badly of me]

.765

Item 如果人们看到我焦虑, 他们会对我失望 [If people

12 see me anxious, they'll put me down]

Item 如我不说一些有趣的事情, 人们就不会喜欢我 [If I

13 don't say something interesting, people won't like me]

Item 如果我不把所有事情弄好, 我就会受到排斥 [If I

10 don't get everything right, I'll be rejected]

Item

15

我需要被所有人喜欢 [I need to be liked by everyone]

.995

Item 我必须得到所有人的认可 [I must get everyone's

11 approval]

$\%$ of Variance $26.88 \% \quad 33.76 \% \quad 5.77 \%$

3 Note. $\mathrm{UB}=$ Unconditional beliefs about self; $\mathrm{CB}=$ Conditional beliefs concerning social

4 evaluation; $\mathrm{HSB}=$ High standards for social performance. 


\section{Table 2 (on next page)}

Table 2 Goodness-of-fit Indices for Confirmatory Factor Analysis. $\left(n_{2}=330\right)$ 
1 Table 2

2 Goodness-of-fit Indices for Confirmatory Factor Analysis. $\left(n_{2}=330\right)$

\begin{tabular}{rcccc}
\hline & \multicolumn{5}{c}{ Goodness-of-fit Indices } \\
& CFI & TLI & RMSEA & $90 \%$ CI \\
\hline Three-factor Model & .961 & .935 & .079 & {$[.055, .104]$} \\
Two-factor Model & .854 & .785 & .171 & {$[.150, .193]$} \\
Single Factor Model & .740 & .636 & .222 & {$[.202,243]$} \\
\hline
\end{tabular}

3

4 


\section{Table 3(on next page)}

Table 3 Invariance Test Across Gender of NSBI. $\left(n_{2}=330\right)$

Note. Model One $=$ configural model; Model Two $=$ equal loadings model; Model Three $=$ equal loadings + intercepts model; Model Four $=$ equal loadings + intercepts + means model. 
1 Table 3

2 Invariance Test Across Gender of NSBI. $\left(n_{2}=330\right)$

\begin{tabular}{lcccccc}
\hline & $\chi^{2}$ & $d f$ & CFI & $\mid \Delta$ CFI $\mid$ & RMSEA & $\mid \Delta$ RMSEA $\mid$ \\
\hline Gender Group & & & & & & \\
Model One & 64.958 & 34 & .965 & - & .074 & - \\
Model Two & 72.588 & 39 & .962 & .003 & .072 & .002 \\
Model Three & 81.425 & 44 & .958 & .004 & .072 & .000 \\
Model Four & 93.211 & 47 & .948 & .010 & .077 & .005
\end{tabular}

$3 \overline{\text { Note. } \text { Model One }=\text { configural model; Model Two }=\text { equal loadings model; Model Three }=\text { equal }}$

4 loadings + intercepts model; Model Four $=$ equal loadings + intercepts + means model.

5 


\section{Table 4(on next page)}

Table 4 Pearson Correlations between the NSBI and Other Anxiety Related Scales. $\left(n_{3}=\right.$ 155)

Notes. $\mathrm{UB}=$ Unconditional beliefs about self; $\mathrm{CB}=$ Conditional beliefs concerning social evaluation; HSB = High standards for social performance; LSAS = Liebowitz Social Anxiety Scale. $* p<.05 ; * * p<.01 ;$. 
$1 \quad$ Table 4

2 Pearson Correlations between the NSBI and Other Anxiety Related Scales. $\left(n_{3}=155\right)$

\begin{tabular}{lcccc}
\hline & HSB & CB & UB & NSBI \\
\hline State Anxiety & .129 & $.212^{* *}$ & $.336^{* *}$ & $.269^{* *}$ \\
Trait Anxiety & $.169^{*}$ & $.196^{*}$ & $.357^{* *}$ & $.286^{* *}$ \\
LSAS & .052 & $.198^{*}$ & $.220^{* *}$ & $.189 *$ \\
\hline
\end{tabular}

3 Notes. $\mathrm{UB}=$ Unconditional beliefs about self; $\mathrm{CB}=$ Conditional beliefs concerning social

4 evaluation; HSB = High standards for social performance; LSAS = Liebowitz Social Anxiety

5 Scale.

$6 \quad * p<.05 ; * * p<.01$

7 


\section{Table 5 (on next page)}

Table 5 Pearson Correlations between the NSBI and Psychological Outcomes. $\left(n_{4}=163\right)$

Notes. UB = Unconditional beliefs about self; $\mathrm{CB}=$ Conditional beliefs concerning social evaluation; HSB $=$ High standards for social performance. ${ }^{*} p<.01$. 


\section{$1 \quad$ Table 5}

2 Pearson Correlations between the NSBI and Psychological Outcomes. $\left(n_{4}=163\right)$

\begin{tabular}{lcccc}
\hline & HSB & CB & UB & NSBI \\
\hline Flourishing & -.108 & $-.239^{* *}$ & $-.303^{* *}$ & $-.261^{* *}$ \\
Anxiety & $.272^{* *}$ & $.235^{* *}$ & $.273^{* *}$ & $.308^{* *}$ \\
Depression & $.241^{* *}$ & $.299^{* *}$ & $.296^{* *}$ & $.334^{* *}$ \\
Stress & $.300^{* *}$ & $.384^{* *}$ & $.348^{* *}$ & $.414^{* *}$
\end{tabular}

3 Notes. $\mathrm{UB}=$ Unconditional beliefs about self; $\mathrm{CB}=$ Conditional beliefs concerning social

4 evaluation; HSB $=$ High standards for social performance.

$5 * * *<.01$.

6 


\section{1}

Figure 1. Confirmatory Factor Analysis Model of the Negative Self-Beliefs Inventory with Standardized Path Coefficients.

Note. $u b=$ Unconditional beliefs about self; $c b=$ Conditional beliefs concerning social evaluation; $\mathrm{hsb}=$ High standards for social performance.

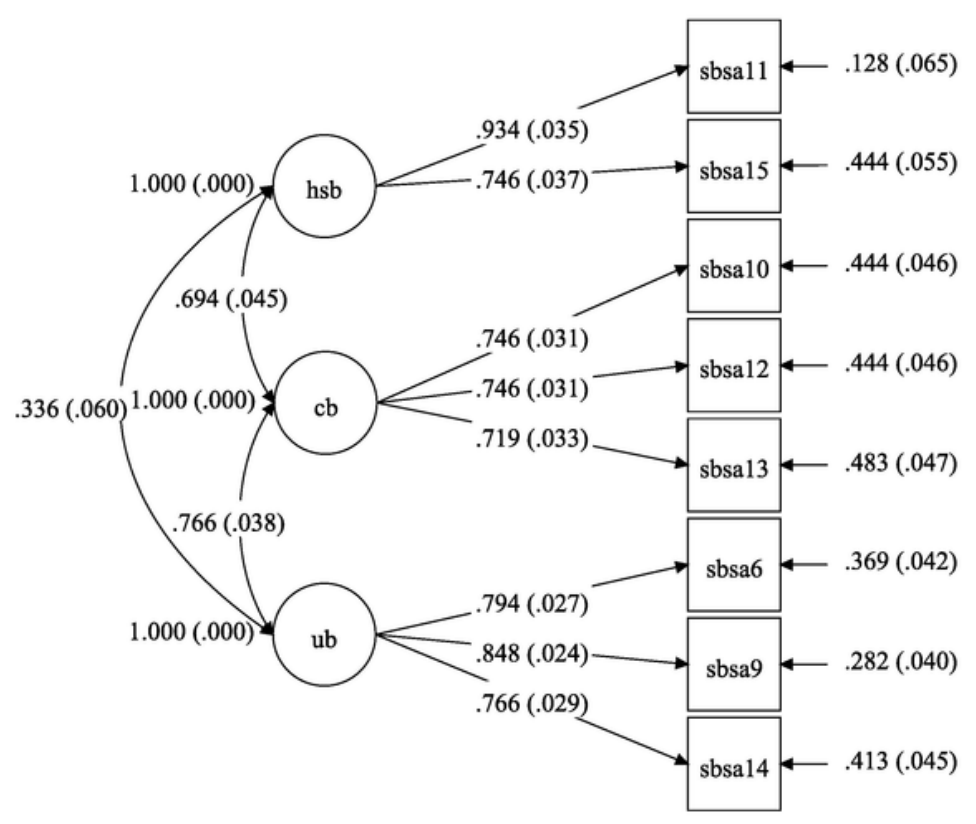

